\title{
Effects of Transverse Baffle Design on Reducing Liquid Sloshing in Partially Filled Tank Vehicles
}

\author{
Xue-lian Zheng, Xian-sheng Li, Yuan-yuan Ren, Yu-ning Wang, and Jie Ma \\ College of Traffic, Jilin University, Changchun 130022, China \\ Correspondence should be addressed to Yuan-yuan Ren; maggie170101@gmail.com
}

Received 17 July 2013; Revised 12 October 2013; Accepted 20 October 2013

Academic Editor: Wuhong Wang

Copyright ( 2013 Xue-lian Zheng et al. This is an open access article distributed under the Creative Commons Attribution License, which permits unrestricted use, distribution, and reproduction in any medium, provided the original work is properly cited.

\begin{abstract}
Longitudinal liquid sloshing in partially filled clear-bore tanks causes extensive degradation of tankers braking performance. To reduce the negative effect of longitudinal liquid sloshing on tankers, three kinds of transverse baffles were designed, namely, the conventional baffle, the circular baffle, and the staggered baffle. Each kind of baffle took several forms to investigate the impact of baffle installation angle, the sizes of holes pierced on the baffle, and their arrangement on the antisloshing effect. FLUENT software was used to simulate liquid sloshing in tanks equipped with different kinds of transverse baffles and subject to constant braking deceleration. A time-series analysis of the forces act on tank walls and transverse baffles was carried out. It was drawn that the baffle shape and its installation angle have great impact on the antisloshing effect of baffles. The study on the antisloshing effect of different transverse baffles is of great significance for tank vehicle driving and braking safety, as well as for the design of optimal transverse baffles.
\end{abstract}

\section{Introduction}

Tank vehicles are widely used in the road transportation of liquid cargoes throughout the world. According to the 2007 Commodity Flow Survey, in the United States, about 1200 million tons of hazardous fluid materials were shipped by tankers per year [1]. In China, about $76 \%$ of chemical products were delivered by tank trucks, and the total freight load was nearly 1 million tons. Tankers are hugely convenient for transporting liquid cargoes and boosting national economic development. However, many traffic accidents occurred, causing losses in terms of human life and property. In 2011, 416 tanker accidents occurred in China, causing 643 deaths and costing tens of millions of Yuan. On the basis of the reported highway accidents involving heavy vehicles, it has been suggested that tank trucks were 4.8 times more likely to be involved in rollovers [2]. Due to the particularity of transportation cargoes, most of the liquid release tanker accidents made direct contamination to water, air, and land $[3,4]$.

A lot of work has been done to investigate the characteristics of tanker accidents. Based on the accident cause, researchers divided them into the following accident types, namely, rollover, rear-end, crash, and over-speeding. Furthermore, the frequencies for each cause were about $45.16 \%$, $25.81 \%, 17.74 \%$, and $11.29 \%$, respectively [5]. Hence, rollover and rear-end are the main forms of tanker accidents.

There are many reasons for tanker accidents [6], but liquid sloshing in partially filled tanks is the most common one. Due to the difference on cargo density and the limitations on vehicle axle load, tank vehicles tend to be operated in the partially filled condition for the majority of the time $[7,8]$. Both the existence of sloshing space and the flow characteristic of liquid cargo make contribution to liquid sloshing when vehicle driving state changes. For moving tanks, a minor change in the vehicle driving state may result in a gigantic surge in the liquid, bringing about liquid sloshing and a large change in the amplitude of the dynamic load $[7,8]$. In addition, the free surface oscillation of a low-viscosity liquid will last for a long time. While tank vehicles commonly subject to intense driver operations in practice, which make the liquid sloshing in partially filled tanks quite violent, the vehicle's handling stability and braking performance are degraded greatly.

Through experimental discovery and theoretical analysis, it was found that tank geometry and liquid fill percentage are 
the main factors impacting the liquid dynamic load transfer, the oscillation amplitude, and the sloshing duration $[9,10]$. To limit liquid sloshing in the longitudinal direction during vehicle braking and acceleration, transverse baffles were designed and have been proved to be quite efficient. To date, the research on transverse baffles can be summarized into the following two aspects.

(1) How do transverse baffle reduce liquid sloshing? To solve this problem, various numerical models have been developed to describe liquid sloshing in tanks with and without transverse baffles [11-15]. Usually, a rectangular tank was chosen as the research object. The fluid fill level was constant while the height of the vertical baffle increased gradually. For vertical baffles of different heights, snap shots of fluidfree surface profiles and elevations, flow patterns, streamlines, and pressure distributions were studied at the same external excitation [11-13]. It has been found that a vortex is originated near the baffle tip and a flow separation is generated before and behind the baffle when liquid sloshing occurs [3]. Moreover, the vortex becomes weaker as the baffle height increases. Compared with liquid sloshing in a clean-bore tank, the gradient of the liquid free surface and the maximum height that the fluid can reach on the tank wall were decreased in tanks with vertical baffles. This leads to smaller movements in the center of gravity of liquid bulk and the enhancement of vehicle braking and acceleration performance.

(2) The influence of the number, appearance, baffle arrangement, and the number and location of holes pierced in the baffle on the antisloshing effect, as in [16-25]. FLUENT simulation and experiment were utilized in this kind of research. The researchers designed kinds of transverse baffle to evaluate their antisloshing effect, including conventional, round, solid-dished, oblique, perforated, and spiral baffles [17-20]. In the simulation process, the liquid fill level was assumed to be constant (50\% was the most popular fill level used) while braking deceleration changes. A time-series analysis of the forces on the tank walls was carried out to investigate the effect of the baffle in terms of reducing liquid sloshing. For all the work that has been carried out on this subject, three problems should be mentioned: First of all, most of the baffles were designed arbitrarily, and national standards on baffle design were not considered. Secondly, the baffle effect on reducing liquid sloshing at different fill levels were not considered yet, while in practice different containers have quite different liquid fill levels due to difference on liquid density and the limitations on road axle load. Thirdly, only sloshing forces on the tank walls were paid attention to, and force analysis of the baffles was not mentioned at all.

Therefore, the purpose of this paper is to design different forms of transverse baffle based on the current Chinese standards then investigate which one has the optimal effect on reducing liquid sloshing. To achieve this purpose, three kinds of transverse baffle were designed and several forms of each kind were used to investigate the impact of the installation angle, the sizes of holes, and their arrangement on the antisloshing effect. Then, FLUENT was used to simulate liquid sloshing in partially filled tanks that are subject to constant braking deceleration, and a time-series analysis of the forces act on the tank walls and the baffles was carried out considering structural damage to both the tank and the baffles.

\section{Materials and Methods}

Tanks with a circular cross-section have greater volumes but the same surface area, which makes them lighter. Therefore, they are the most popular and were used in this paper. Three kinds of baffle were designed and FLUENT was used to simulate longitudinal liquid sloshing in partially filled tanks with a circular cross-section and equipped with baffles.

2.1. Theory of Similarity. The hydrodynamic equations are solved by FLUENT using a finite-volume method. In this method, the 2- or 3-dimensional tank model is split into thousands of meshes at first and then the hydrodynamic equations are solved in each of them. Therefore, the bigger the tank model is, the more time the simulation or calculation procedure needs. For time saving, the full-sized tank was scaled down to a tank model.

The criteria of geometric similarity, kinematic similarity, and dynamic similarity must be satisfied to ensure that liquid sloshing in the full-sized tank and that in the tank model exhibit similar hydrodynamic behavior. The full-sized tank is given the subscript $t$ and the tank model $m$. According to the geometric similarity, the length-scale factor can be calculated as follows:

$$
\delta_{L}=\frac{L_{t}}{L_{m}}
$$

where $L$ is the geometric size of the tank.

The velocity-scale factor that expresses the relation between the moving speed of the full-sized tank and that of the tank model can be expressed by the following equation:

$$
\delta_{v}=\frac{v_{t}}{v_{m}}
$$

where $v$ is the moving velocity of the tank.

In general, speed can be expressed as the differential of distance, which is expressed as follows:

$$
v=\frac{d L}{d t} .
$$

Therefore, according to (2) and (3), the velocity-scale factor can be reexpressed as follows:

$$
\delta_{v}=\frac{v_{t}}{v_{m}}=\frac{d L_{t} / d t_{t}}{d L_{m} / d t_{m}}=\frac{d L_{t}}{d L_{m}} \frac{d t_{m}}{d t_{t}}=\delta_{L} \delta_{t} .
$$
$(4)$ :

Then, the time-scale factor can be written as follows using

$$
\delta_{t}=\frac{\delta_{L}}{\delta_{v}} .
$$

The acceleration-scale factor is drawn by differentiating velocity and is presented as follows:

$$
\delta_{a}=\frac{\delta_{v}^{2}}{\delta_{L}}
$$


For longitudinal liquid sloshing in partially filled tank vehicles, forces due to gravity and inertia play a more important role than the viscous and turbulent shear forces. Therefore, the Froude number, which is the function of gravity and inertia, is the most relevant dimensionless parameter for liquid sloshing, which is expressed as follows:

$$
F_{r}=\frac{v_{t}^{2}}{L_{t} g_{t}}=\frac{v_{m}^{2}}{L_{m} g_{m}} .
$$

For the situation discussed in this paper,

$$
g_{t}=g_{m},
$$

and the following equations can be deduced:

$$
\begin{aligned}
\frac{v_{t}^{2}}{v_{m}^{2}} & =\frac{L_{t}}{L_{m}}, \\
\delta_{v}^{2} & =\delta_{L} .
\end{aligned}
$$

Therefore, some important conclusions can be derived from (10), (5), and (6) and are presented as follows:

$$
\begin{gathered}
\delta_{v}=\sqrt{\delta_{L}}, \\
\delta_{t}=\sqrt{\delta_{L}}, \\
\delta_{a}=1 .
\end{gathered}
$$

Equations in (11) are the conditions that must be satisfied to ensure that liquid sloshing in the tank model exhibits similar dynamic behavior to that in the full-sized tank.

While the liquid dynamic behavior in both the full-sized tank and the tank model are similar, the longitudinal sloshing force obtained by liquid sloshing simulation in the tank model is proportionate to that obtained in the full-sized tank. To derive the relationship of the two forces, the momentum for the longitudinal liquid sloshing is needed, which is calculated as follows:

$$
\frac{\partial}{\partial t} \int_{\tau} \rho \omega d \tau+\oint_{A} \rho \omega(\vec{v} \vec{n}) d A=\sum F_{z},
$$

where $\tau$ is the control volume, $\omega$ is the velocity of the control volume along the $z$ direction, $\rho$ is liquid's density, $A$ is the control plane, $\vec{v}$ is the velocity of the control volume, and $\vec{n}$ is the normal of the vertical plane.

In the case where fluid can only flow into the control volume through one of the control planes and flow out through another one, (12) can be simplified as follows:

$$
Q_{m}\left(\omega_{2}-\omega_{1}\right)=\sum F_{z}
$$

where $Q_{m}$ is the fluid discharge in the control volume; $\omega_{2}$ is fluid flow-out velocity; $\omega_{1}$ is fluid flow-in velocity.

Suppose that the time interval is quite small; then (13) can be rewritten as follows:

$$
\Delta Q_{m} \Delta \omega=\Delta F_{z} .
$$

TABLE 1: Parameter values of the full-sized tank and the tank model.

\begin{tabular}{lcc}
\hline & $\begin{array}{c}\text { The full-sized } \\
\text { tank }\end{array}$ & $\begin{array}{c}\text { The tank } \\
\text { model }\end{array}$ \\
\hline Length & $8 \mathrm{~m}$ & $2 \mathrm{~m}$ \\
Radius of circular cross section & $1.1 \mathrm{~m}$ & $0.275 \mathrm{~m}$ \\
Height of the elliptical head & $0.55 \mathrm{~m}$ & $0.1375 \mathrm{~m}$ \\
Longitudinal deceleration & $-3 \mathrm{~m} / \mathrm{s}^{2}$ & $-3 \mathrm{~m} / \mathrm{s}^{2}$ \\
\hline
\end{tabular}

The transient flow rate is generally expressed by

$$
\Delta \omega=\frac{\Delta Q_{m}}{A d} .
$$

Therefore, (14) can be reexpressed as follows:

$$
(\Delta \omega)^{2} d A=\Delta F_{z} .
$$

According to (16), the ratio of the longitudinal sloshing force generated in the full-sized tank to that in the tank model in the same situation can be expressed as follows:

$$
\delta_{F}=\frac{\Delta F_{t}}{\Delta F_{m}}=\frac{\left(\Delta \omega_{t}\right)^{2} d A_{t}}{\left(\Delta \omega_{m}\right)^{2} d A_{m}}=\delta_{v}^{2} \delta_{A}=\delta_{L} \delta_{L}^{2}=\delta_{L}^{3} .
$$

In other words, the ratio is $1: \delta_{L}^{3}$.

2.2. Baffle Design. The dimensions and other variables of the full-sized tank and the tank model are listed in Table 1. The length-scale factor is set at 4 . The two ends of the tank are covered by $2: 1$ elliptical heads. According to the current Chinese standard, GB/T 25198-2010 Heads for pressure vessels, the height of the head is just a quarter of the diameter and its radius varies between the major and minor axis.

In real life, the braking deceleration of a heavy truck can be expressed as follows:

$$
a_{x}=g \varphi,
$$

where $a_{x}$ is the vehicle's longitudinal deceleration and $\varphi$ is the road adhesion coefficient.

For heavy trucks, the coefficient of road adhesion is about 0.68. Therefore, the maximum longitudinal deceleration of tank trucks is approximately 6.7. In this paper, a deceleration of 3 is chosen for the tank model.

Before baffle design, the current national standard in China should be considered. The current Chinese standard, GB/T 18564-2001 General specification for normal pressure tank body of transportation liquid dangerous goods, states the following.

(1) The transverse baffle should be capable of bearing the following longitudinal inertia force:

$$
F_{x}=m \times 2 g,
$$

where $m$ is the mass of liquid bulk.

(2) The free area of the transverse baffle should be greater than $40 \%$ of the tank's cross-sectional area. For tanks 
with a volume less than or equal to $25 \mathrm{~m}^{3}$, the volume between the two tank cross-sections at which adjacent baffles locate should be less than or equal to $3 \mathrm{~m}^{3}$; for those with a volume greater than $25 \mathrm{~m}^{3}$, the volume between adjacent cross-sections can be up to $7 \mathrm{~m}^{3}$.

(3) A manhole should be cut in the center of the baffle, and the top and bottom segments should be removed to allow air and fluid, respectively, to drain out.

For this paper, three kinds of baffle have been designed according to the national standard, namely, conventional, circular, and staggered baffles. The baffles are designed to fit the geometric size of the tank model.

2.2.1. The Conventional Baffle. The conventional baffle is depicted in Figure 1. Its free area is set to comprise 57.34\% of the tank's cross-sectional area. The removed top segment comprises $15 \%$ and the removed bottom segment $27.66 \%$ of tank's cross-sectional area in order to enable liquid to drain away quickly and to play the role of manhole at the same time.

To investigate the influence of the baffle installation angle on liquid sloshing reduction, different oblique angles are configured, as shown in Figure 2. The oblique angle is set to be 0 -, 5-, 10-, 15-, and 20-degree. The corresponding baffles are labeled N0, N5, N10, N15, and N20. It should be noted that all of the conventional baffles have the same projection area in the vertical plane.

2.2.2. The Circular Baffle. A series of circular baffles have been designed to investigate how the sizes of pierced holes influence the liquid sloshing reduction, as shown in Figure 3. The free area of all the circular baffles is set to comprise $57.34 \%$ of the tank's cross-sectional area. The removed top segment is equal to $5 \%$ of cross-sectional area and the removed bottom segment $10 \%$ in order to enable liquid to drain away quickly.

In Figure 3(a), only a manhole is cut in the center of the baffle. In Figures 3(b)-3(e), the radius of the manhole is decreased and a few small holes are arranged around it. The circular baffles plotted in Figures 3(a)-3(e) are labeled C0, C2, C4, C6, and C18, respectively, according to the number of small holes.

2.2.3. The Staggered Baffle. Unlike the conventional and circular baffle, a complete staggered baffle is divided into two parts, and the free area of each part is set to be $28.67 \%$ of the tank's cross-sectional area. The two parts look just the same, as shown in Figure 4. The baffle has the same radius as the tank's cross-section so as to the baffle match with the tank well. The two baffle parts are located in two vertical planes, and there is a longitudinal distance between them.

The angle from the $X$-coordinate to the line connecting the middle point of the baffle chord and the center of the tank's cross-section is defined as the arrangement angle, as shown in Figures 5-6.

The staggered baffle can be arranged in two ways: either the arrangement angles of the two adjacent baffle parts are opposite to each other, as presented in Figure 5, which is defined as the reverse staggered baffle or the arrangement

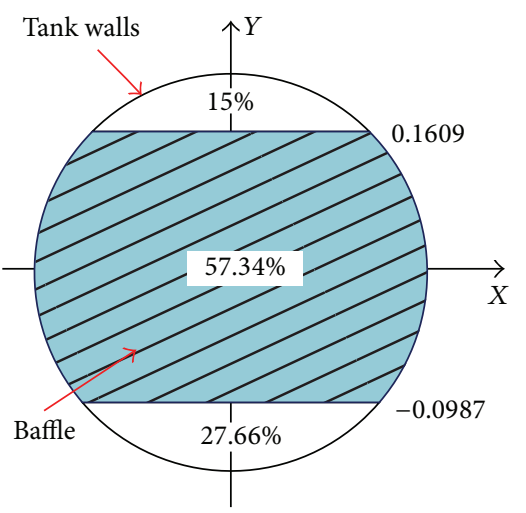

FIgURE 1: Schematic diagram of the conventional baffle.

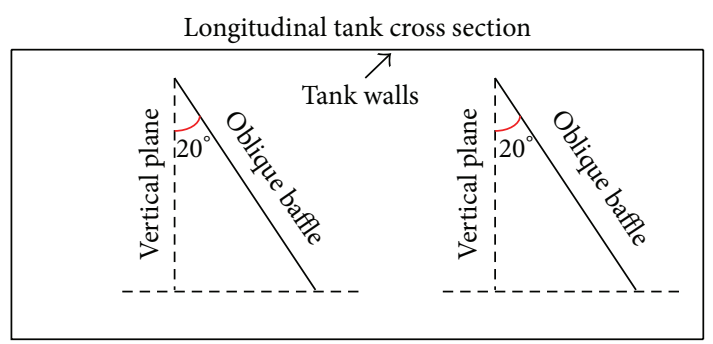

FIGURE 2: Oblique angle of conventional baffles.

angles are equal to one another, as shown in Figure 6, which is defined as the similar staggered baffle. For each option, the absolute value of the arrangement angle is set as 10-, 15-, and 20-degree in this paper, and labeled SR10, SR15, and SR20 (reverse staggered baffles) and SS10, SS15, and SS20 (similar staggered baffles).

2.2.4. The Spatial Distribution of Baffles. The volume between the two adjacent tank cross-sections at which baffles locates should meet the specified requirements. For the conventional and circular baffle, four baffles are needed, and they divide the tank into five parts, as illustrated in Figure 7. Four groups of staggered baffle made up of eight baffle parts are needed, and they divide the tank into nine parts, as Figure 8 shows.

By the principle of inertia, fluid flows forward when tanker brakes, and the fore elliptical head (the wall marked as wall1 in Figures 7 and 8) bears the maximum sloshing force. To make the illustration of the simulation results easier, the fore and aft tank walls as well as the baffles are all labeled, as shown in Figures 7 and 8.

2.3. FLUENT Simulation Settings. Before liquid sloshing is simulated using FLUENT, tank models equipped with the designed baffles were constructed and meshed. To save on simulation time and ensure simulation accuracy at the same time, the interval size of the mesh volume was set at 0.05 units.

The volume-of-fluid (VOF) method was utilized to simulate the two-phase (liquid and air) flow phenomenon. The reference pressure location was arranged at any spatial point 


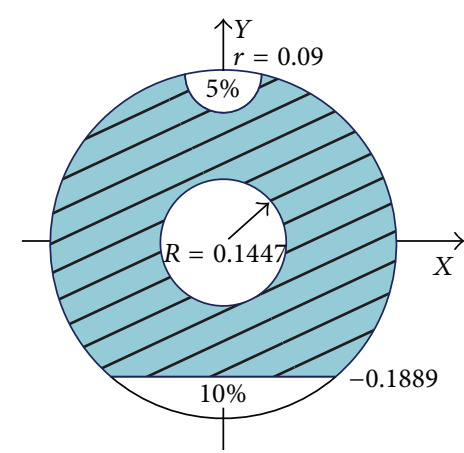

(a) The circular baffle with a central manhole, "C0"

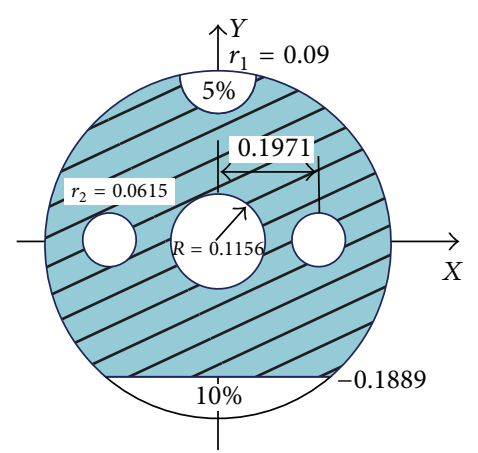

(b) The circular baffle with a central manhole and two small holes, "C2"

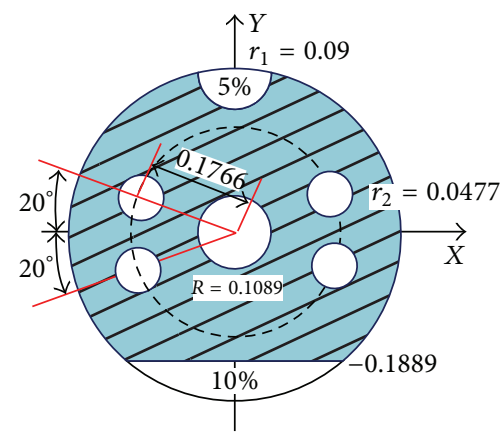

(c) The circular baffle with a central manhole and four small holes, "C4"

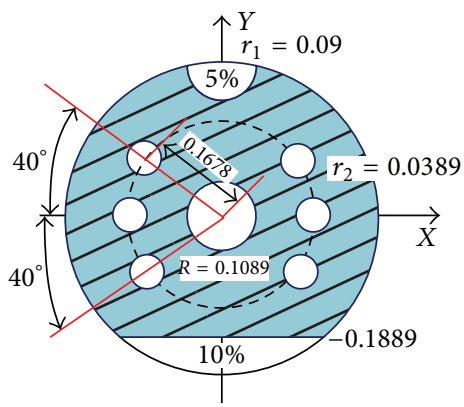

(d) The circular baffle with a central manhole and six small holes, "C6"

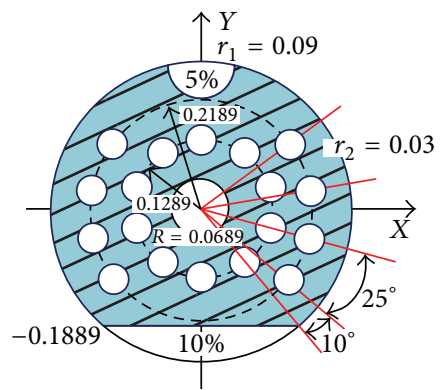

(e) The circular baffle with a central manhole and eighteen small holes, "C18"

FIGURE 3: A series of circular baffles.

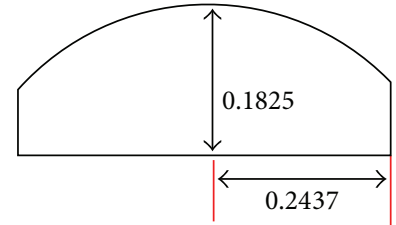

FIgURE 4: A part of a staggered baffle.

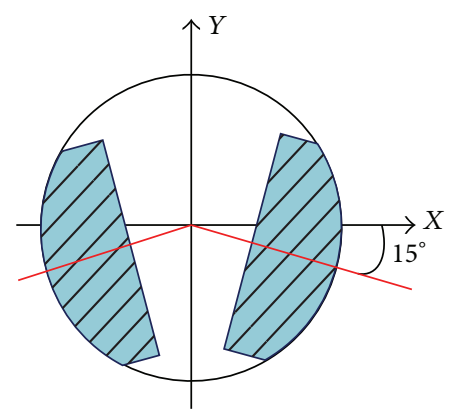

FIGURE 5: The reverse staggered baffle.

outside of the tank to ensure that the simulation reflects the actual situation accurately.

Unlike other research, deceleration was applied to the tank continuously instead of being withdrawn at a certain time. Supposing that the initial braking speed is $80 \mathrm{~km} / \mathrm{h}$,

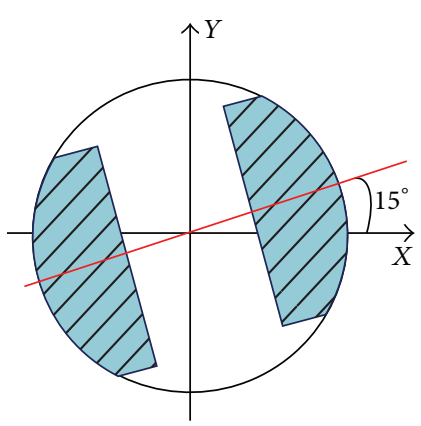

Figure 6: The similar staggered baffle.

while the maximum braking deceleration the road can provide is about $6.7 \mathrm{~m} / \mathrm{s}^{2}$, the truck needs 3-4 seconds to stop completely. Generally, the simulation time for liquid sloshing is about 2 seconds, which is less than the actual braking time. Therefore, if the simulation time is not being increased, there is no need to withdraw the deceleration during the simulation, and a full-development liquid sloshing process can be monitored and studied.

According to market research, in practice, the liquid fill percentage or fill level is in the range of $0.4-0.8$ most of the time. Therefore, the liquid fill level varied from 0.4 to 0.8 with a step size of 0.1 in this paper.

During the simulation, the longitudinal sloshing forces on all of the walls were monitored separately. 


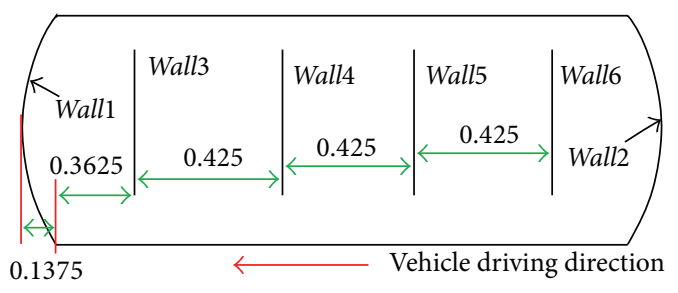

FIGURE 7: The distribution of the conventional and circular baffle.

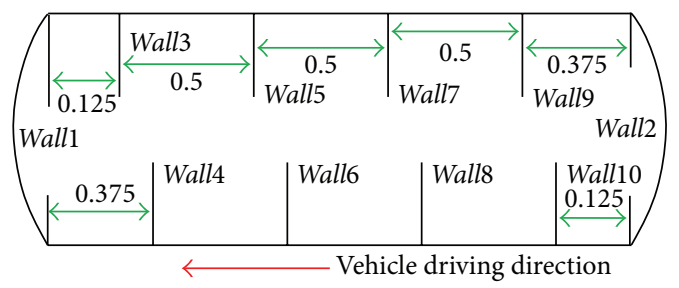

FIgURE 8: The distribution of the staggered baffle.

\section{Results and Discussion}

Liquid sloshing in tanks without baffles was simulated first and the maximum sloshing force on wall1 was compared with the result obtained using the quasistatic method to verify the accuracy of the FLUENT simulation settings.

In the quasistatic method, the longitudinal sloshing force on wall 1 is expressed as follows:

$$
F_{x}=m a_{x}
$$

As the liquid fill level changes from 0.4 to 0.8 with a 0.1 step-size, the fluid volume (fluid in the elliptical heads is included) takes the following values: $0.7975 \mathrm{~m}^{3}, 0.9083 \mathrm{~m}^{3}$, $1.0713 \mathrm{~m}^{3}, 1.1987 \mathrm{~m}^{3}$, and $1.3616 \mathrm{~m}^{3}$.

The results of the comparison were presented in Figure 9. It is known that liquid sloshing forces obtained using the quasistatic method are a little smaller than those obtained by FLUENT simulation. While liquid sloshing obtained using the quasistatic method is very close to the actual, the comparison result reveals the correctness of FLUENT simulation settings.

For each kind of baffle, several forms existed. Therefore, an intergroup comparison of the influence of different baffle forms on the liquid sloshing reduction was carried out first. Then a comparison of the best baffle forms for each kind was made to determine the largest limitation to the liquid sloshing.

3.1. Results of the Conventional Baffle. The longitudinal sloshing forces on wall1-wall6 in tanks equipped with conventional baffles and with the 0.4 liquid fill level of 0.4 are plotted in Figure 10.

In Figures 10(a)-10(b), it is quite obvious that N0, whose oblique angle is 0 -degree, has much less of a reducing effect on liquid sloshing than the other baffles. Moreover, the sloshing forces on wall3-wall6 in the tank equipped with N0 are much larger than the forces on the same wall in tanks equipped with

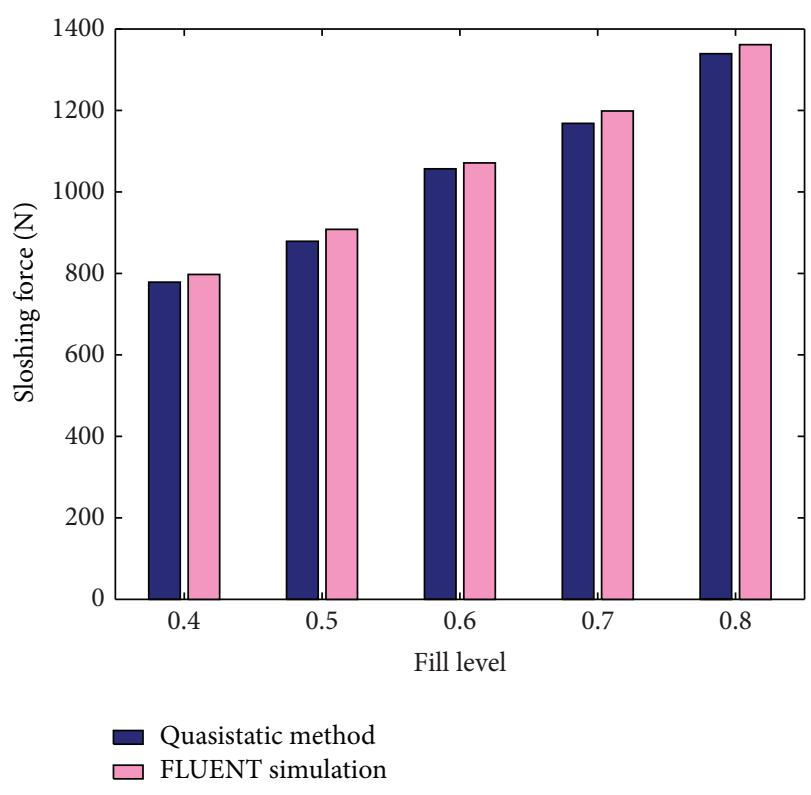

Figure 9: Comparison of the longitudinal liquid sloshing forces obtained using FLUENT simulation and the Quasistatic method.

N5-N20, as shown in Figures 10(c)-10(f). Furthermore, N5$\mathrm{N} 20$ have almost the same effect on liquid sloshing reduction. It is known that the larger the sloshing force on the baffle is, the more easily damaged it will be. Therefore, N0 is the worst choice as a transverse baffle when liquid percentage is 0.4 .

It can be seen in Figure 10 that the sloshing forces on wall 1 and wall 3 are much bigger than those on the other walls. As the scale ratio between the FLUENT simulation sloshing force and the real-life case is 1:64, wall 1 and wall3 should be paid the most attention. Therefore, for liquid fill levels of $0.5-0.8$, only the sloshing forces on wall 1 and wall 3 are presented (see Figure 11). The sloshing force on wall 1 reflects the baffle effect in terms of reducing longitudinal liquid sloshing in partially filled tanks, and the sloshing force on wall 3 is just the maximum sloshing force the baffle bears.

It can be seen in Figures 11(a1)-11(d1) that the sloshing forces on wall 1 differ very little between tanks equipped with conventional baffles of different arrangement angles at the same liquid fill level. The same can be said of the sloshing forces on wall3. That is to say that, at the same liquid fill level, conventional baffles of different oblique angles have almost identical effects in terms of reducing liquid sloshing, and the maximum sloshing forces on the baffles are also quite similar.

However, a closer watch reveals that the following conclusions can be drawn. For liquid fill level changing from 0.5 to $0.6, \mathrm{~N} 0$ does not reduce liquid sloshing to the same extent as the other baffles, and the force on wall3 is also larger than those in tanks equipped with the baffles of N5-N20. For the liquid fill level of 0.7 , the sloshing forces on wall 1 in tanks equipped with conventional baffles of N0-N20 are quite close to each other, so are the sloshing forces on wall3. Finally, for the liquid fill level of 0.8 , N10 bears the largest sloshing force and is not as effective as the other baffles in limiting sloshing. 


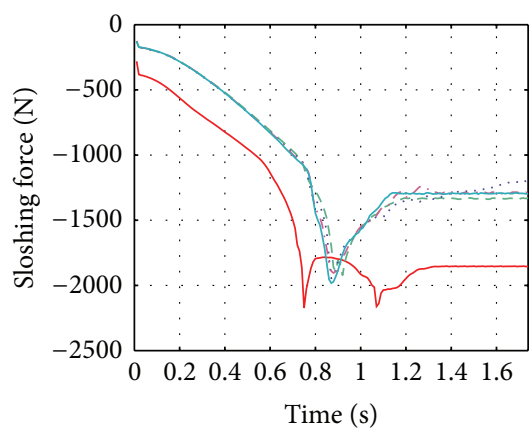

(a) Sloshing force on wall 1

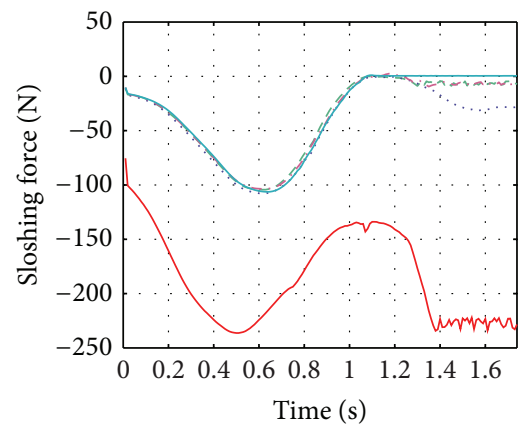

$\begin{array}{llll}- & \text { N0 } & \text { N15 } \\ --- & \text { N5 } & \text { N20 } \\ \ldots \ldots . & \text { N10 } & & \end{array}$

(d) Sloshing force on wall4

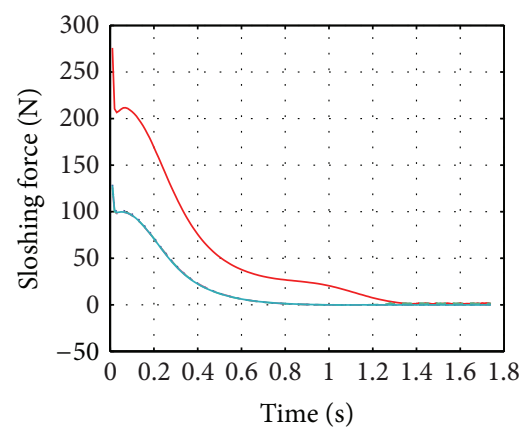

(b) Sloshing force on wall2
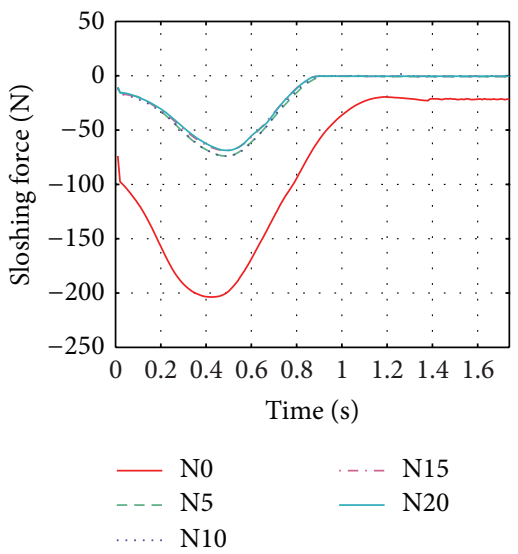

(e) Sloshing force on wall5

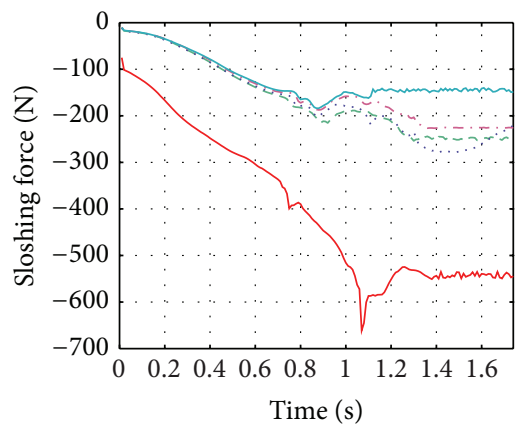

(c) Sloshing force on wall3

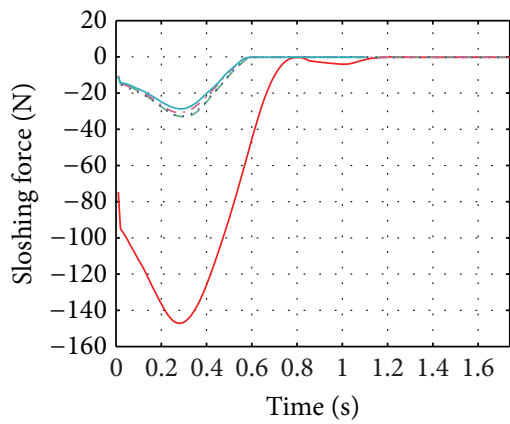

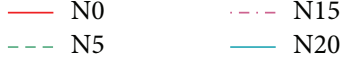

..... $\mathrm{N} 10$

(f) Sloshing force on wall6

FIGURE 10: Sloshing forces on wall1-wall6 in tanks with conventional baffles and the liquid fill level of 0.4.

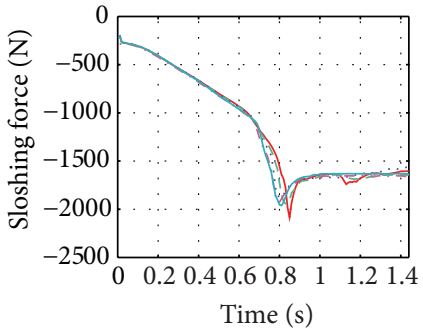

(a1) Sloshing force on wall1

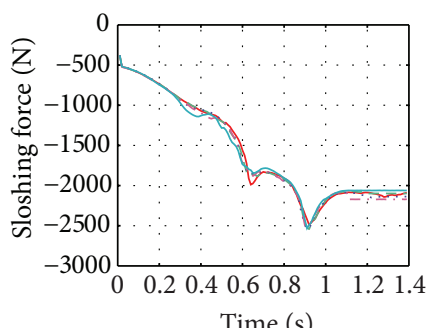

Time (s)

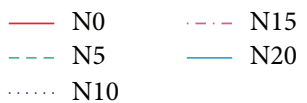

(c1) Sloshing force on wall

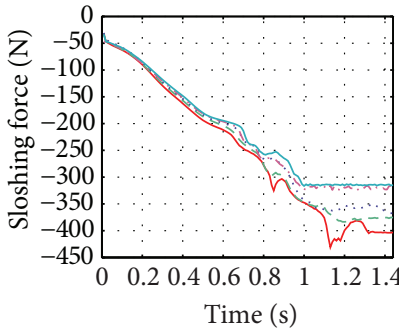

(a2) Sloshing force on wall3
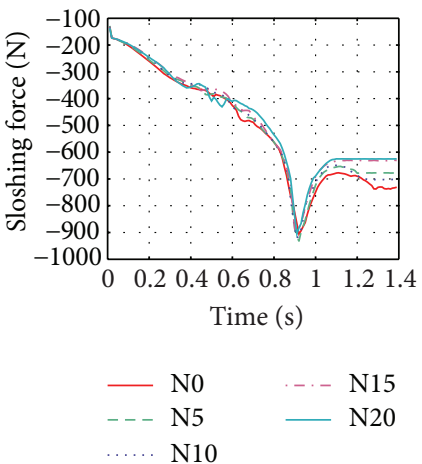

(c2) Sloshing force on wall3

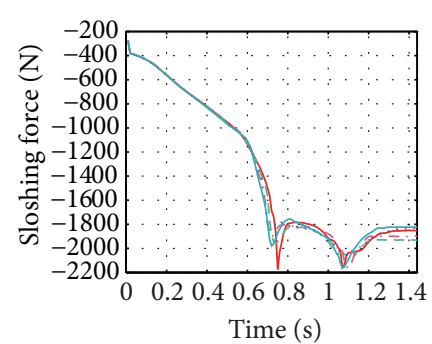

(b1) Sloshing force on wall1
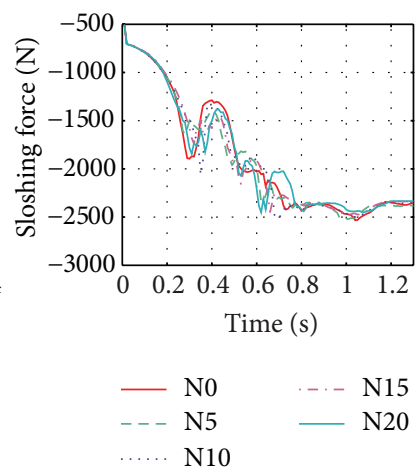

(d1) Sloshing force on wall1

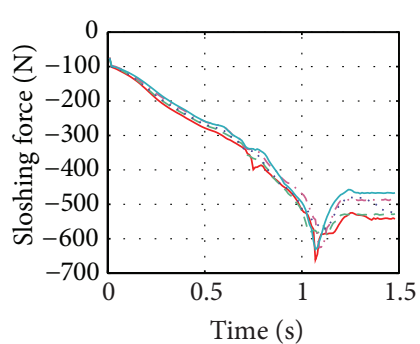

(b2) Sloshing force on wall3

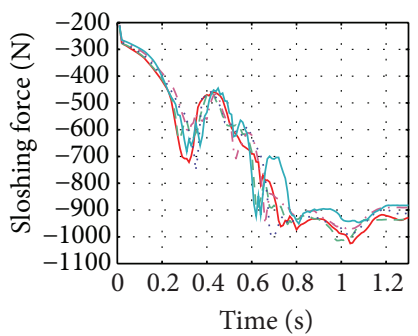

FIGURE 11: Sloshing forces on wall 1 and wall 3 in tanks with conventional baffles and liquid fill levels of 0.5-0.8: (a) fill level = 0.5; (b) fill level = 0.6 ; (c) fill level $=0.7$; (d) fill level $=0.8$. 


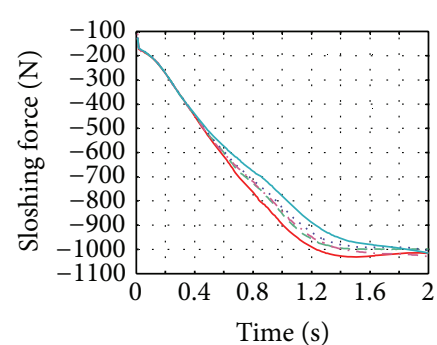

(a1) Sloshing force on wall

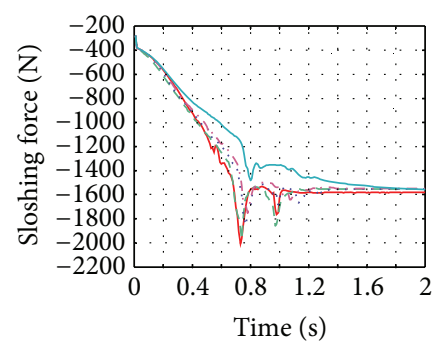

(c1) Sloshing force on wall

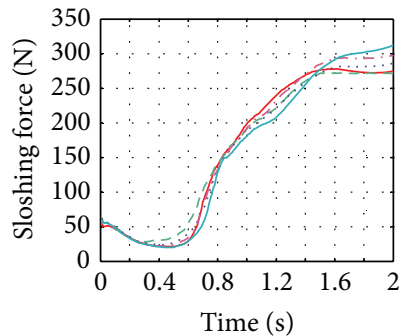

(a2) Sloshing force on wall3

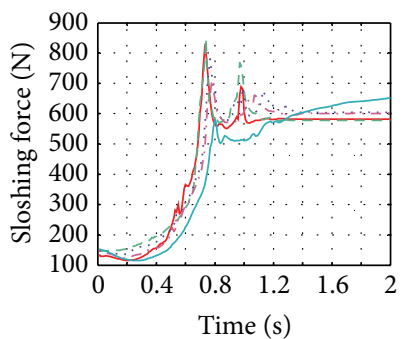

(c2) Sloshing force on wall3

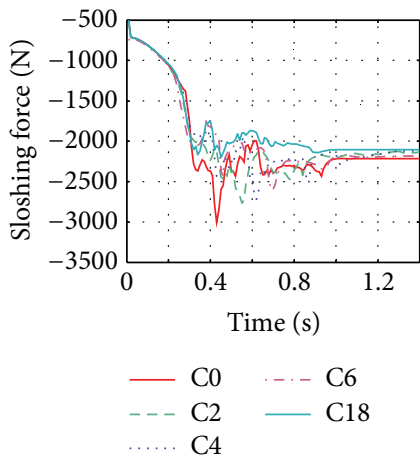

(e1) Sloshing force on wall

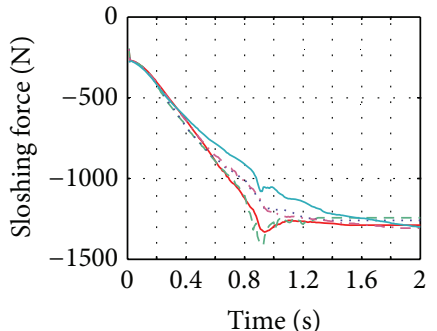

(b1) Sloshing force on wall

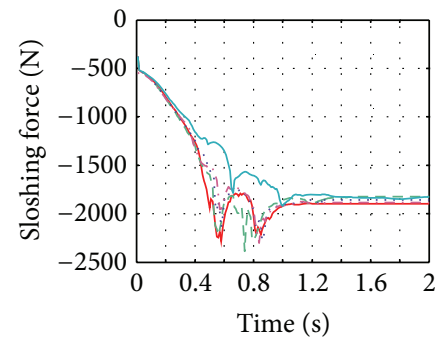

(d1) Sloshing force on wall

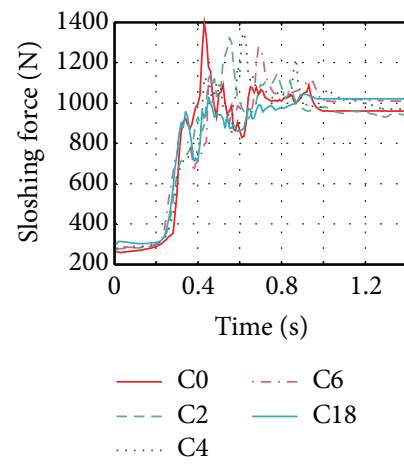

(e2) Sloshing force on wall3

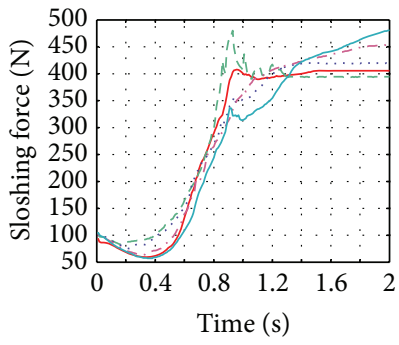

(b2) Sloshing force on wall3

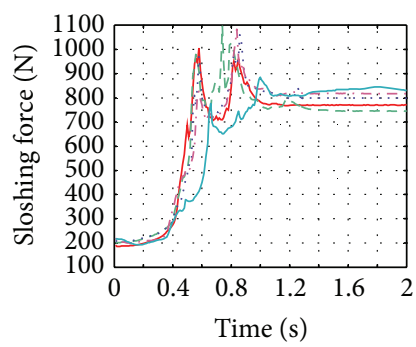

(d2) Sloshing force on wall3

Figure 12: Sloshing forces on wall 1 and wall3 in tanks with circular baffles and liquid fill level changing from 0.4 to 0.8: (a) fill level = 0.4; (b) fill level $=0.5$; (c) fill level $=0.6$; (d) fill level $=0.7$; (e) fill level $=0.8$.

According to the above analysis, N5, N15, and N20 are the ideal conventional baffles as they limit sloshing the most. However, while baffles with different oblique angles have the same projection area in the vertical plane, the larger the oblique angle is, the heavier the baffle will be. That will increase the structural container's mass and reduce the useful load capacity of the tank vehicle. Therefore, N5 is the best conventional baffle.

3.2. Results of the Circular Baffle. The number of circular baffle arranged in the tank model is equal to that of conventional baffle used in the previous section. Similar with the former, the longitudinal sloshing forces on wall2 and wall4-wall6 are much smaller than those on wall 1 and wall3. Therefore, only the sloshing forces on wall1 and wall3 are provided here, again for liquid fill level changing from 0.4 to 0.8 . The simulation results were used to evaluate the sloshing restraint effects of different circular baffles and the maximum sloshing forces the baffles have to bear.

Under liquid sloshing in tanks equipped with different circular baffles, the forces on wall 1 and wall 3 change steadily while the liquid fill level changes from 0.4 to 0.5 , as shown in Figure 12. As the liquid fill level increases, the variation in the forces becomes irregular and harsh, and many sharp peaks and valleys appear. These abrupt changes in the forces acting on the tank walls and the baffles could reduce the lifetimes of both. Therefore, such large and abrupt force change is not welcome. The steadier the force change is, the better force analysis the object has, even if the steady value of the force is a little higher.

It can be seen in Figures 12(a1)-12(e1) that, for circular baffles with the same free area, the smaller the radii of the holes (including both the manhole and the small holes around it) are, the more holes the baffle must have, and thus the larger the reduction in sloshing will be. For the tank equipped with $\mathrm{C} 18$, the sloshing force on wall1 is the smallest, and the force variation on this baffle is much smoother. Therefore, C18, the baffle with a central manhole and eighteen small holes around it has the best effect in terms of reducing liquid sloshing.

The sloshing forces on the baffles tend to steady values with an increase in simulation time. However, the steady sloshing forces on wall 1 and wall 3 in the tank equipped with 


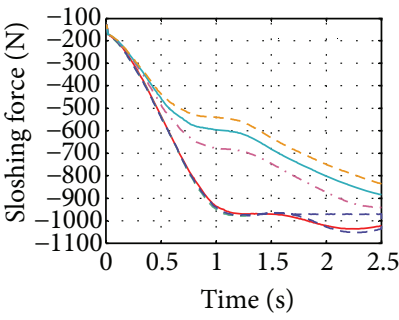

(a) Sloshing force on wall 1

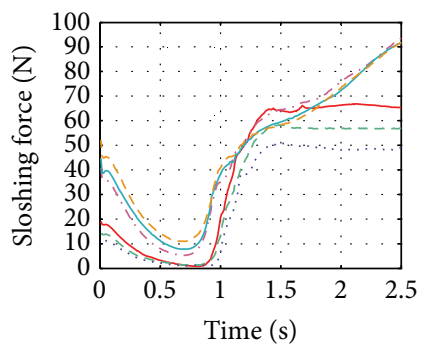

(e) Sloshing force on wall5

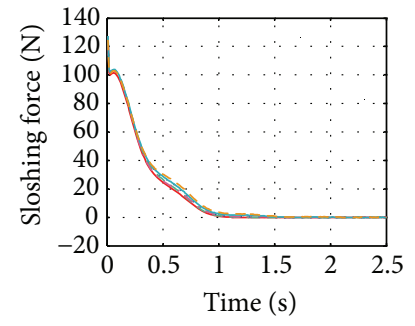

(b) Sloshing force on wall2

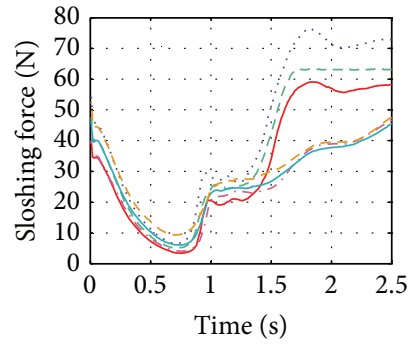

(f) Sloshing force on wall6
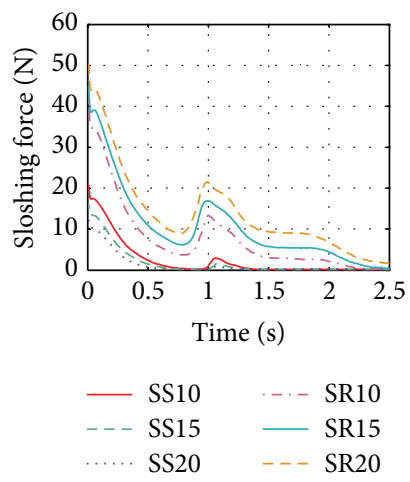

(i) Sloshing force on wall9

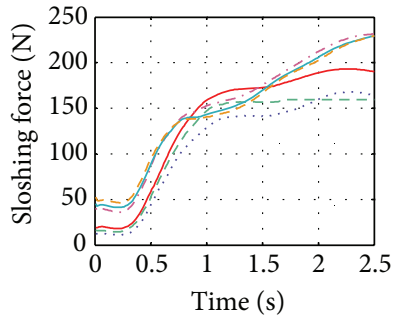

(c) Sloshing force on wall3

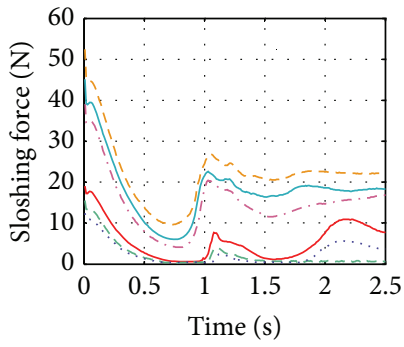

(g) Sloshing force on wall7

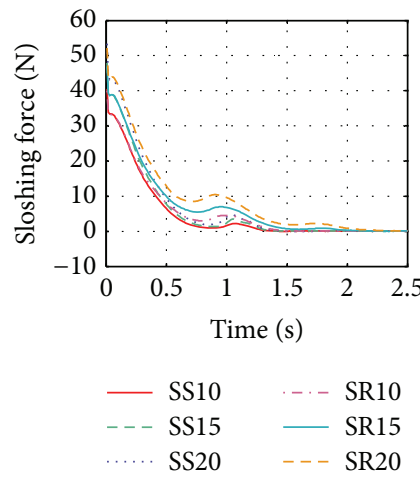

(j) Sloshing force on wall 10

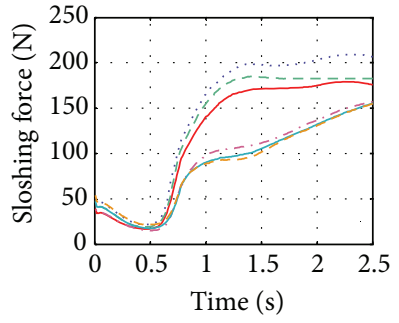

(d) Sloshing force on wall4

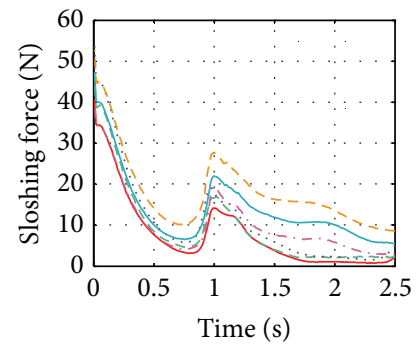

(h) Sloshing force on walls

FIGURE 13: Sloshing forces on wall1-wall10 in tank equipped with staggered baffles and with the liquid fill level of 0.4.

C18 are not the smallest when the liquid sloshing develops fully. As mentioned earlier, while we pay much more attention to the steady and gentle change of sloshing forces, their absolute values are less of importance. Therefore, C18, the baffle with a central manhole and 18 small holes around the manhole, is the best circular baffle.

We can also discover that circular baffle with more holes has a better antisloshing effect. According to the force distribution of the baffle, sloshing forces near the holes are much bigger. By Newton's third law, liquid sloshing force is greatly degraded when liquid flows through the holes. Moreover, the small holes make much bigger contribution to the antisloshing effect. Therefore, if we could get a baffle with many small holes pierced on it, the baffle must have an excellent behavior on reducing liquid sloshing. Limited by mesh-grid size and simulation time, simulation for liquid sloshing in a tank equipped with such baffles is not conducted.

3.3. Results of the Staggered Baffle. Unlike the conventional and circular baffles, a complete staggered baffle is composed of two parts, as shown in Figures 5 and 6. They are arranged in a staggered fashion so as to restrain fluid sloshing in partially filled tank vehicles. Therefore, there are four baffle pieces fitted on the left side of the tank and four on the right, as shown in Figure 8.

In tanks equipped with different staggered baffles and with the liquid fill percentage of 0.4 , the longitudinal sloshing forces on wall1-wall 10 are plotted in Figure 13. It can again be seen that the sloshing forces on wall 1 and wall 3 are much bigger than those on the other walls. As in the cases of the conventional and circular baffles, wall 1 and wall3 bear the biggest sloshing forces and are the most easily damaged parts in the tank.

In Figure 13(a) we can see that SR20 has the best effect in terms of reducing liquid sloshing. However, as can be seen in Figures 13(c), 13(e), and 13(g)-13(j), the sloshing forces on wall 3 , wall5, and wall7-wall 10 in the tank with SR20 are the largest. In this situation, the sloshing restraint effect is chosen as the criterion for evaluating the best staggered baffle. This is due to the fact that sloshing force on wall 1 is much bigger than that on wall3. 


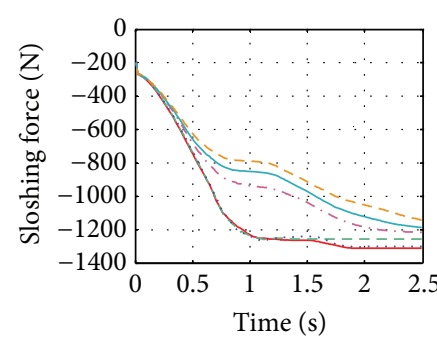

(a1) sloshing force on wall 1
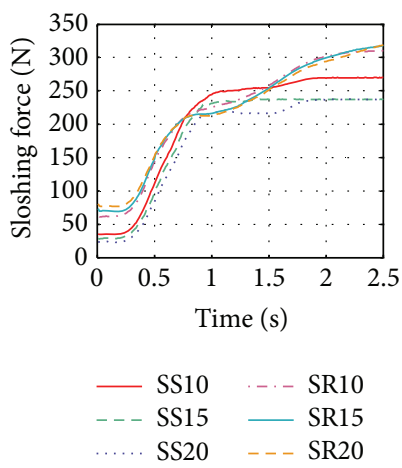

(a2) sloshing force on wall3

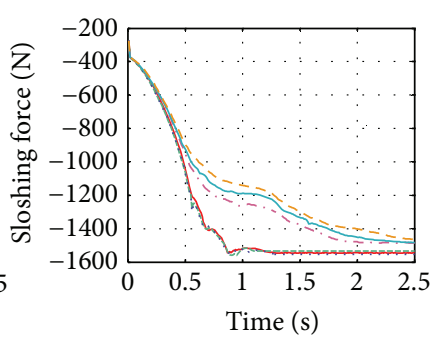

(b1) sloshing force on wall

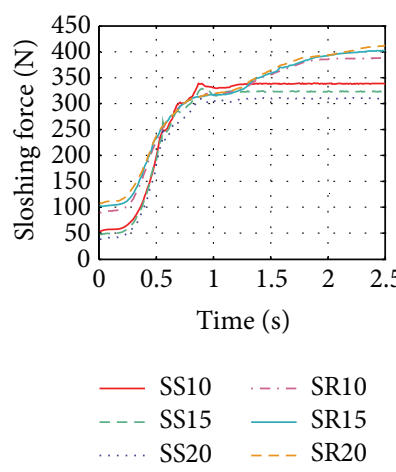

(b2) sloshing force on wall3

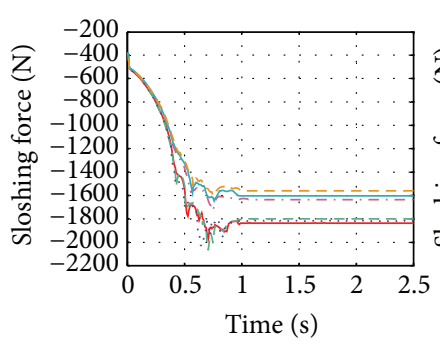

(c1) sloshing force on wall 1
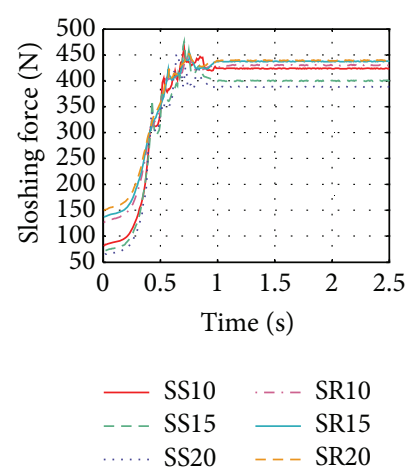

(c2) sloshing force on wall

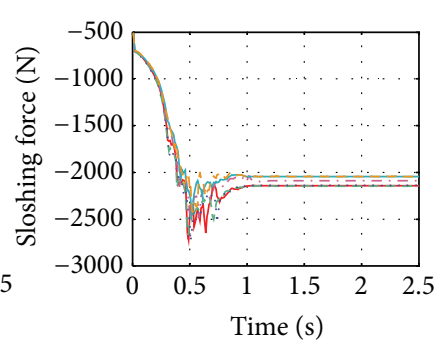

(d1) sloshing force on wall 1
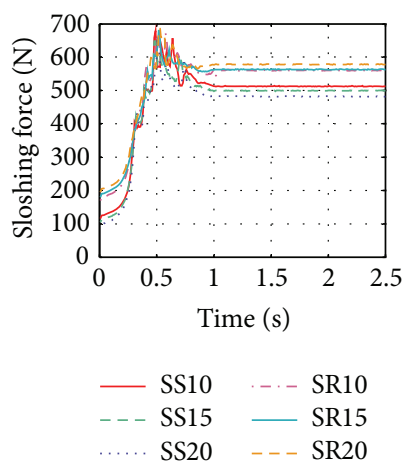

(d2) sloshing force on wall3

FIGURE 14: Sloshing forces on wall 1 and wall 3 in tanks equipped with staggered baffles and with liquid fill levels of 0.5-0.8: (a) fill level = 0.5; (b) fill level = 0.6; (c) fill level $=0.7$; (d) fill level $=0.8$.

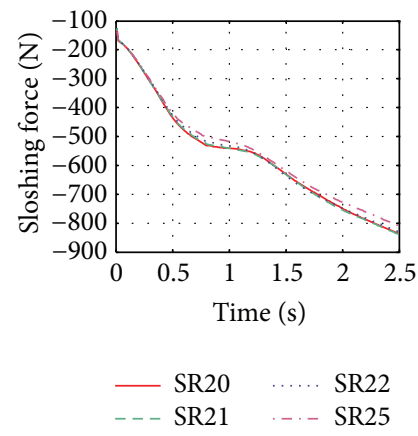

(a) Liquid fill level of 0.4

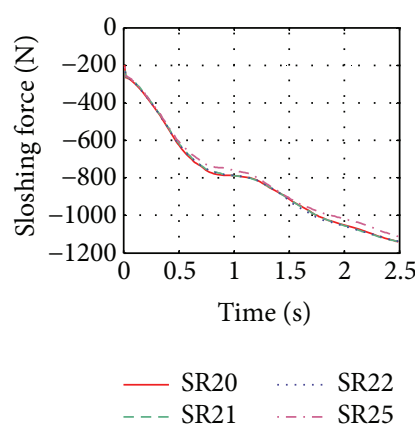

(b) Liquid fill level of 0.5

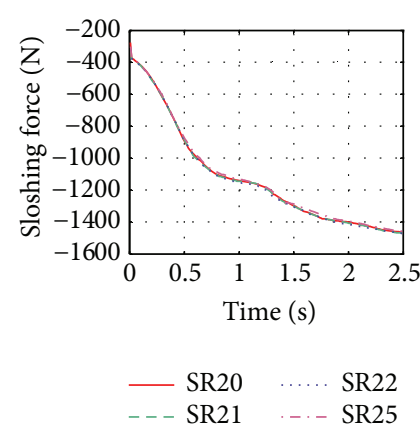

(c) Liquid fill level of 0.6

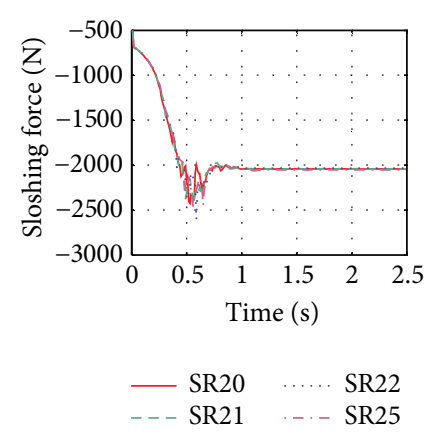

(d) Liquid fill level of 0.8

FIGURE 15: Sloshing forces on wall1 in tanks equipped with reverse staggered baffles and with liquid fill levels of 0.4-0.8.

The sloshing forces on wall 1 and wall 3 in tanks with staggered baffles while the liquid fill level changes from 0.5 to 0.8 are presented in Figure 14. It is very apparent that SR20 has the best effect on reducing the liquid sloshing, and the SR20 baffles bear the largest sloshing forces at the same time. As the fill level increases, the sloshing forces on the walls become irregular and harsh, and force peaks and valleys appear. The variations in the sloshing forces generated in the tank equipped with the SR20 baffle are much smoother than those generated in tanks equipped with the other staggered baffles. Therefore, the SR20 baffle can be considered as the best the staggered baffle.

It can be drawn in Figures 14(a1)-14(d1) that, for the reverse staggered baffle, there is a direct relationship between the arrangement angle and the baffle's antisloshing effect.
With the increase of arrangement angle, the antisloshing effect of the baffle becomes better. Consequently, a few more reverse staggered baffles whose arrangement angles are 21degree, 22-degree, and 25-degree were designed to investigate whether SR20 is indeed the best staggered baffle, and the complementary reverse staggered baffles are labeled SR21, SR22, and SR25. An arrangement angle that is bigger than 25degree was not considered to ensure that the fluid could still drain away quickly and easily.

Therefore, for tanks equipped with reverse staggered baffles whose arrangement angle is 20-, 21-, 22- and 25-degree, the sloshing forces on wall1 while liquid fill level changing from 0.4 to 0.8 are plotted in Figure 15. As the curves on wall 1 were quite similar to fill levels of 0.7 and 0.6 , only the curves for the liquid fill level of 0.6 are shown. 


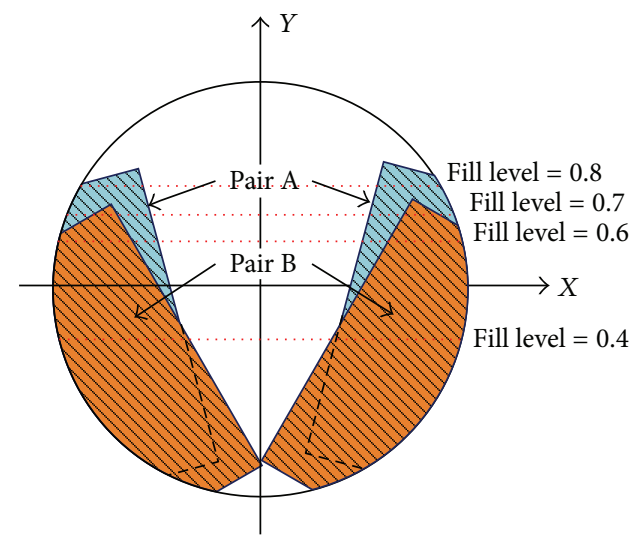

FIGURE 16: Schematic diagram for the antisloshing effect of staggered baffles.

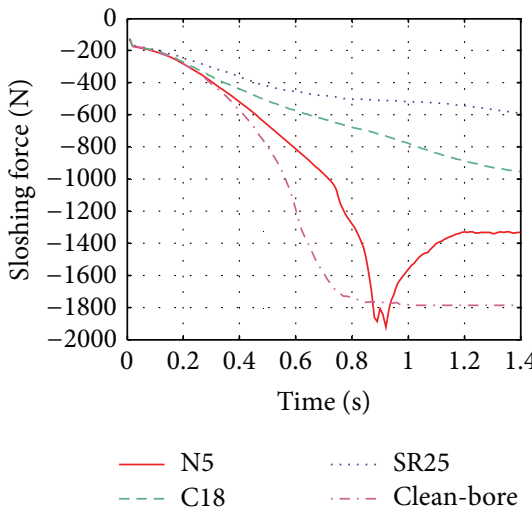

(a) Liquid fill level of 0.4

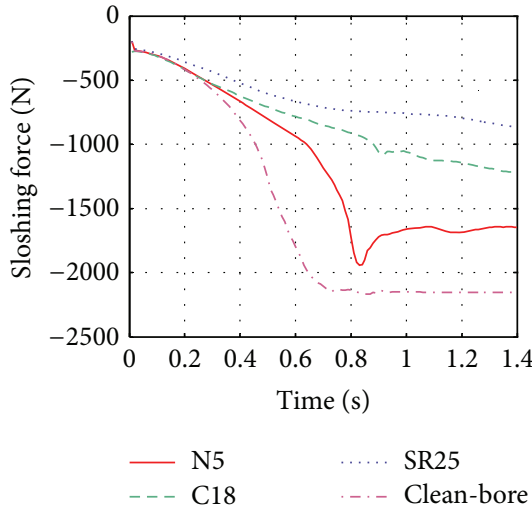

(b) Liquid fill level of 0.5

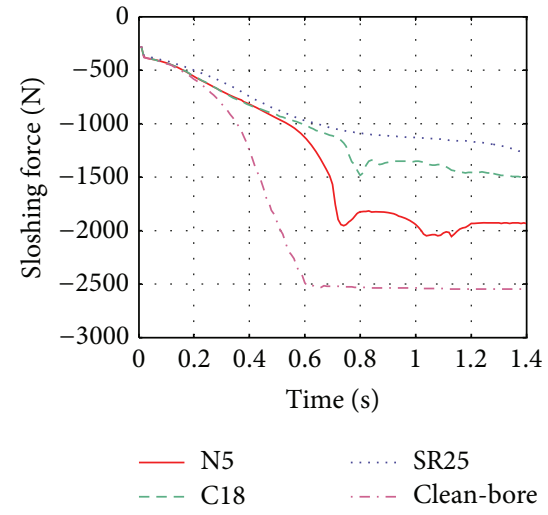

(c) Liquid fill level of 0.6

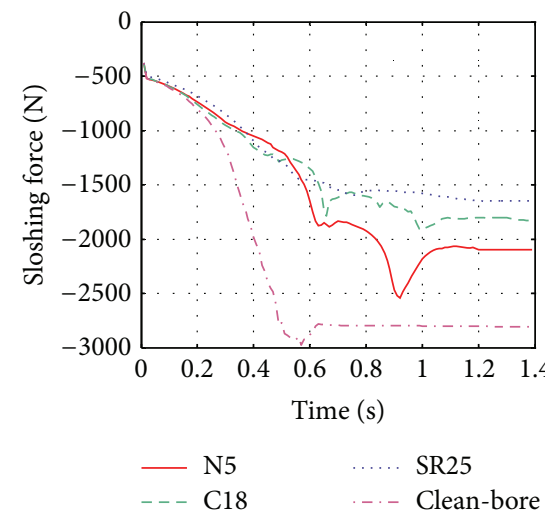

(d) Liquid fill level of 0.7

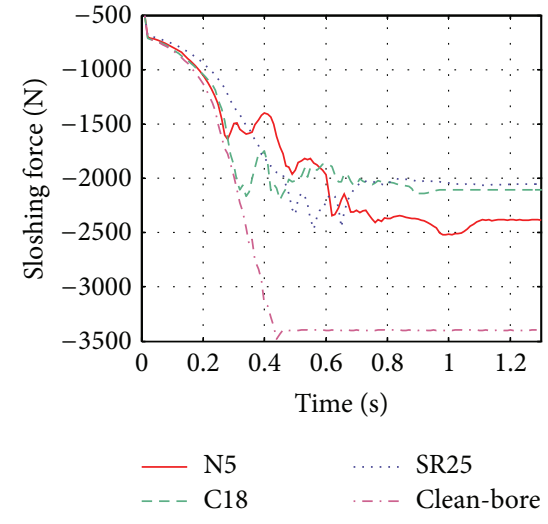

(e) Liquid fill level of 0.8

FIGURE 17: Sloshing forces on wall1 in tanks equipped with different kinds of baffles and with liquid fill levels of 0.4-0.8.

The increase in the arrangement angle strengthens the reduction effect of the reverse staggered baffle on liquid sloshing. However, for sloshing forces generated in tanks with the reverse staggered baffles of 20- and 25-degree arrangement angle, with the increase of the liquid fill level, the difference in these two cases becomes smaller. Furthermore, the change in the baffle's sloshing restraint effect when the baffle arrangement angle change from 20- to 25-degree is smaller than that when the baffle arrangement angle changes from 5- to 20-degree (under the same conditions). While it is not suggested that the arrangement angle should exceed 25-degree, it can be deduced that the reverse staggered baffle with an arrangement angle of 25-degree is the best staggered baffle.

An attempt was made to discover why the bigger the arrangement is, the better antisloshing effect the staggered baffle has. With the increase in the arrangement angle, for liquid fill level changes from 0.4 to 0.8 , the area of staggered baffles used for reducing liquid sloshing grows, as shown 
in Figure 16. While the external acceleration or deceleration keeps constant, the bigger the antisloshing area is, the better antisloshing effect the baffle has.

3.4. Effect of Different Kinds of Baffles on the Reduction of Liquid Sloshing. Now, the three baffles with the best liquid sloshing restraint effects, one of the conventional baffle, one of the circular baffle, and one of the staggered baffle, are compared to each other so as to select the most effective one. The comparison curves are plotted in Figure 17.

It is obvious that the reverse staggered baffle with an arrangement angle of 25-degree has much more better effect on reducing liquid sloshing than the other baffles when the liquid fill level is in the range of 0.4-0.7. When the liquid fill level is higher than $0.7, \mathrm{C} 18$, the circular baffle with a central manhole and eighteen small holes gives an identical performance to the latter. The conventional baffle performs quite poorly when the liquid fill level is smaller than 0.7. Therefore, across the whole range of liquid fill levels, the reverse staggered baffle with an arrangement angle of 25-degree would be the first choice for reducing liquid sloshing in a tank with a circular cross-section.

\section{Conclusions}

To reduce longitudinal liquid sloshing in a partially filled tank, the conventional, circular, and staggered baffles were deigned and several forms in each kind were used to investigate the impact of baffle installation angle, the sizes of holes in the baffle, and their arrangement on the antisloshing effect. A full-sized tank was scaled down to a tank model according to the theory of similarity to save on simulation time. The FLUENT software was used to simulate liquid sloshing in tanks with different fill percentages, equipped with kinds of baffles and subject to constant braking deceleration. A time-series analysis on forces that act on tank walls and transverse baffles was carried out to obtain the viable optimal baffle designed in this paper.

It was found that the fore elliptical head and the baffle closest to the fore head are the most easily damaged parts in a partially filled tank equipped with baffles. Therefore, sloshing forces on wall 1 and wall 3 must be examined to ensure the structural integrity of both the tank and the baffle. By a time-series analysis of the sloshing forces, it was found that for the conventional baffle, baffle equipped with a 5-degree oblique angle is the best one on reducing liquid sloshing, and the sloshing force on this baffle is the smallest. For the circular baffle, baffle has a central manhole and 18 small holes around it is the most effective one on reducing liquid sloshing, and the changing of forces on this baffle is much smoother. For staggered baffles, the reverse staggered baffle with an arrangement angle of 25-degree has the best antisloshing effect; however, the force on this baffle is the biggest. In the end, by comparison of the three baffles that are chosen from each kind of baffle, the staggered baffle is the optimal one on reducing liquid sloshing.

During the analysis procedure, it was discovered that the baffle area used for antisloshing has a direct relation with baffle's liquid sloshing reduction effect. The bigger the area used for antisloshing is, the better antisloshing effect the baffle has. Besides, the distance between adjacent baffles can make great contribution to the development of liquid sloshing; hence, a smaller distance is suggested. Combined with the limitation on the structural container's mass, the reverse staggered baffle might be the best transverse baffle on reducing liquid sloshing.

Since we just illustrate liquid sloshing in tanks with different transverse baffles, the function mechanism of transverse baffle on antisloshing as a further study should be conducted.

\section{Conflict of Interests}

The authors declare that there is no conflict of interests regarding the publication of this paper.

\section{Acknowledgments}

The research is supported by Research on Evaluation and Detection Technology of Handling and Driving Stability for Commercial Vehicles (no. 2009BAG13A04), the National Natural Science Foundation of China (Grant no. 51375200), and the National Natural Science Foundation of China (Grant no. 51208225).

\section{References}

[1] Research and Innovative Technology/Bureau of Transportation Statistics, "Transportation-2007 commodity flow survey," 2010.

[2] J. Woodrooffe, "Evaluation of dangerous goods vehicle safety performance," Transport Canada Report, TP 13678 E, 2000.

[3] C. Kwon, "Conditional value-at-risk model for hazardous materials transportation," in Proceeding of the Winter Simulation Conference (WSC '11), pp. 1703-1709, Phoenix, Ariz, USA, December 2011.

[4] L. E. Ghosh, S. M. Rapik, and P. L. B. Christopher, "Release risk model to evaluate highway cargo tank component-specific safety performance in transporting hazardous materials," in Proceeding of the 91st Annual Meeting Transportation Research Board (TRP '12), Washington, DC, USA, 2012.

[5] K. Z. Liu, H. X. Liu, Y. C. Yan et al., "Analysis of the road accidents with the tank-vehicle transportation of hazardous materials," Journal of Safety and Environment, vol. 21, no. 2, pp. 18-21, 2012.

[6] W. Wang, W. Zhang, H. Guo, H. Bubb, and K. Ikeuchi, "A safetybased approaching behavioural model with various driving characteristics," Transportation Research Part C, vol. 19, no. 6, pp. 1202-1214, 2011.

[7] M. I. Salem, H. Victor, M. Mucino et al., "Review of parameters affecting stability of partially filled heavy-duty tankers," in Proceeding of the SAE International, Truck \& Bus Meet Exposition, 1999-01-3709, Detroit, Mich, USA, November 1999.

[8] X. Kang, S. Rakheja, and I. Stiharu, "Cargo load shift and its influence on tank vehicle dynamics under braking and turning," The International Journal of Heavy Vehicle Systems, vol. 9, no. 3, pp. 173-203, 2002.

[9] F. Solaas and O. M. Faltinsen, "Combined numerical and analytical solution for sloshing in two-dimensional tanks of general 
shape," Journal of Ship Research, vol. 41, no. 2, pp. 118-129, 1997.

[10] K. Modaressi-Tehrani, S. Rakheja, and I. Stiharu, "Threedimensional analysis of transient slosh within a partly-filled tank equipped with baffles," Vehicle System Dynamics, vol. 45, no. 6, pp. 525-548, 2007.

[11] T. Kandansamy, S. Rakheja, and A. K. W. Ahmed, "An analysis of baffles designs for limiting fluid slosh in partly filled tank trucks," The Open Transportation Journal, vol. 4, pp. 23-32, 2010.

[12] D. Liu and P. Lin, "Three-dimensional liquid sloshing in a tank with baffles," Ocean Engineering, vol. 36, no. 2, pp. 202-212, 2009.

[13] H. Akyildiz, "A numerical study of the effects of the vertical baffle on liquid sloshing in two-dimensional rectangular tank," Journal of Sound and Vibration, vol. 331, no. 1, pp. 41-52, 2012.

[14] J. R. Cho and H. W. Lee, "Numerical study on liquid sloshing in baffled tank by nonlinear finite element method," Computer Methods in Applied Mechanics and Engineering, vol. 193, no. 2326, pp. 2581-2598, 2004.

[15] N. Kang and K. Liu, "Influence of baffle position on liquid sloshing during braking and turning of a tank truck," Journal of Zhejiang University: Science A, vol. 11, no. 5, pp. 317-324, 2010.

[16] A. Dasgupta, Effect of tank cross-section and longitudinal baffles on transient liquid slosh in partly-filled road tankers [M.S. thesis], Department of Mechanical and Industrial Engineering, Concordia University, 2009.

[17] N. Lloyd, E. Vaiciurgis, and T. A. G. Langrish, "The effect of baffle design on longitudinal liquid movement in road tankers an experimental investigation," Transactions of the Institution of Chemical Engineers, Part B, vol. 80, no. 4, pp. 181-185, 2002.

[18] M. A. Xue, J. H. Zheng, and P. Z. Lin, "Numerical simulation of sloshing phenomena in cubic tank with multiple baffles," Journal of Applied Mathematics, vol. 2012, Article ID 245702, 21 pages, 2012.

[19] K. Threepopnartkul and C. Suvanjumrat, "effect of baffles on fluid sloshing inside the moving rectangular tanks," in Proceeding of the 2nd TSME International Conference on Mechanical Engineering, Krabi, Thailand, October 2011.

[20] K. Modaressi-Tehrani, S. Rakheja, and I. Stiharu, "Role of transverse baffle designs on transient three-dimensional liquid slosh in a partly-filled circular tank," in Proceeding of the SAE Commercial Vehicle Engineering Congress and Exhibition, 2005-013594, Chicago, Ill, USA, November 2005.

[21] M. J. Fabela-Gallegos, A. Ramires-Valencia, C. A. FavalaGallegos et al., "Experimental assessment of baffles and their effect on the longitudinal sloshing force in a scaled elliptical tank," in Proceeding of the SAE Commercial Vehicle Engineering Congress and Exhibition, 2005-01-3577, Chicago, Ill, USA, November 2005.

[22] K. Modaressi-Tehrani, S. Rakheja, and I. Stiharu, "Threedimensional analysis of transient slosh within a partly-filled tank equipped with baffles," Vehicle System Dynamics, vol. 45, no. 6, pp. 525-548, 2007.

[23] M. J. Fabela-Gallegos, D. Vazquez-Vega, M. Martinez-Madrid et al., "Experimental evaluation of baffles geometry and their configuration in partially filled scaled elliptical tank," in Proceeding of the SAE Commercial Vehicle Engineering Congress and Exhibition, 2005-01-3581, Chicago, Ill, USA, November 2005.

[24] G. R. Yan, S. Rakheja, and K. Siddiqui, "Baffle design analysis for a road tanker transient fluid slosh approach," SAE International Journal of Commercial Vehicles, 2008-01-2670, 2008.
[25] I. M. Ibrahim, "Anti-slosh damper design for improving the roll dynamic behavior of cylinder tank trucks," in Proceeding of the SAE International Truck and Bus Meeting and Exposition, 199901-3729, Detroit, Mich, USA, November 1999. 


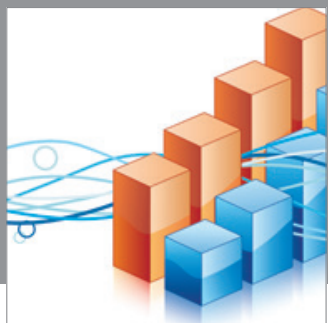

Advances in

Operations Research

mansans

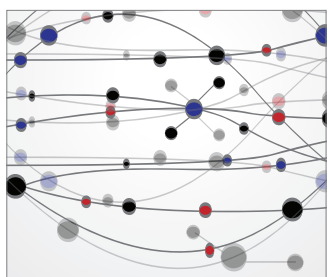

The Scientific World Journal
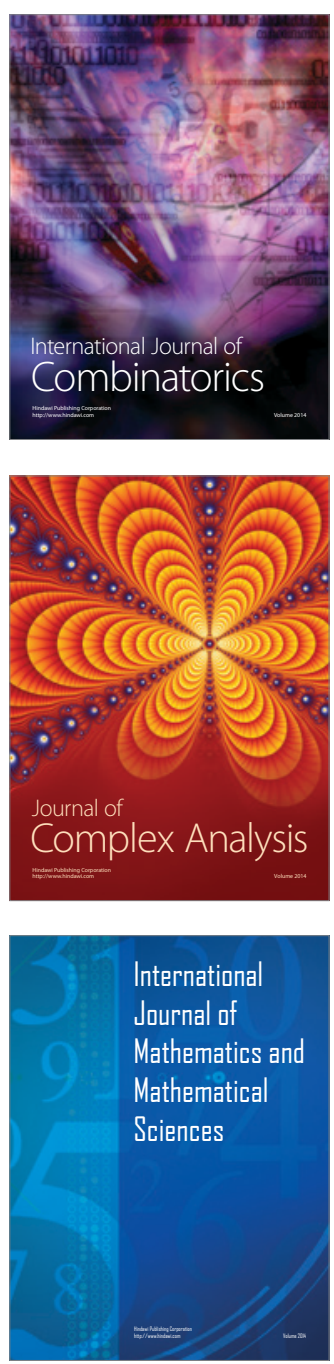
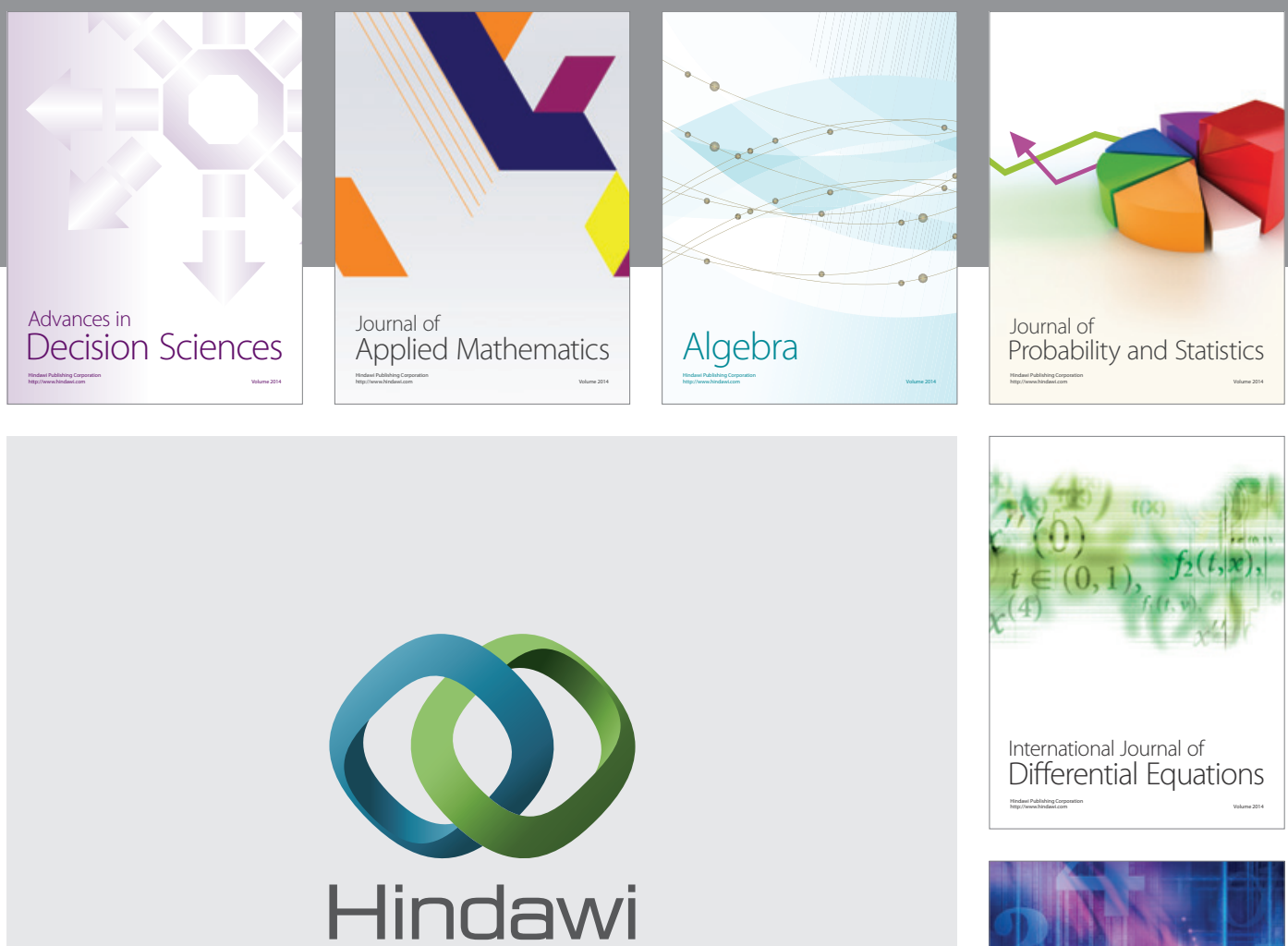

Submit your manuscripts at http://www.hindawi.com
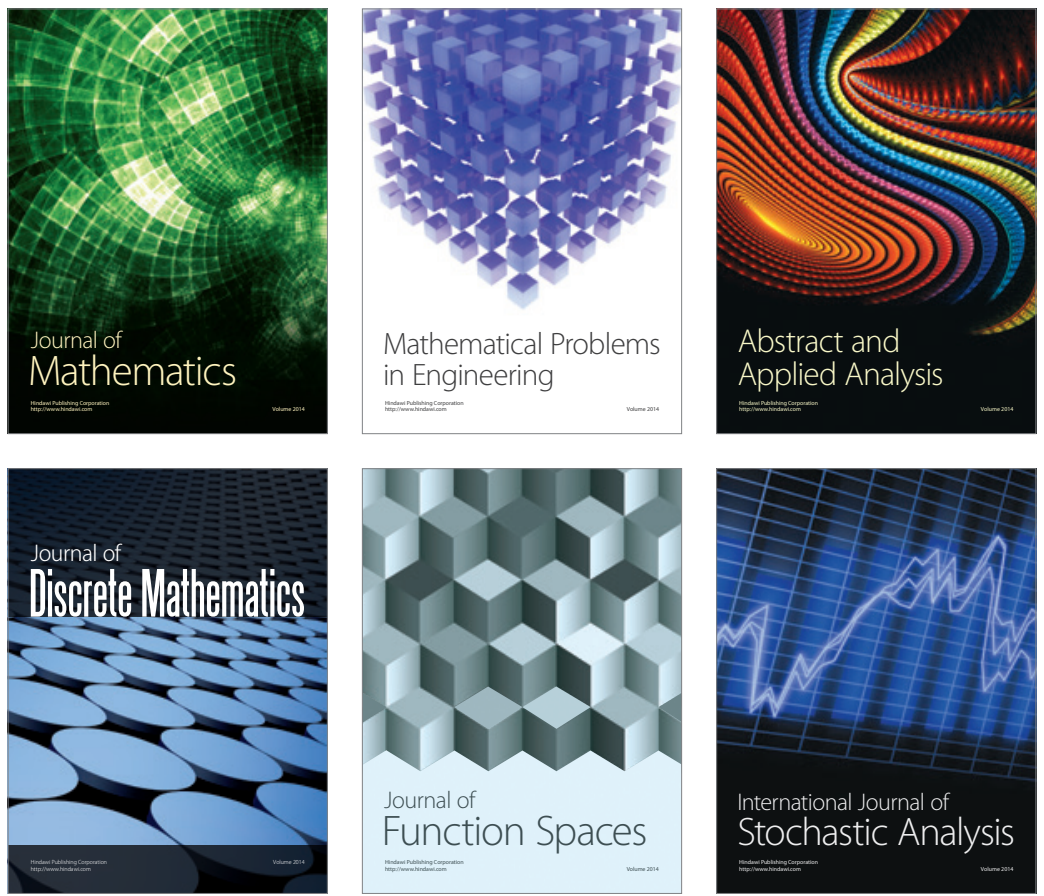

Journal of

Function Spaces

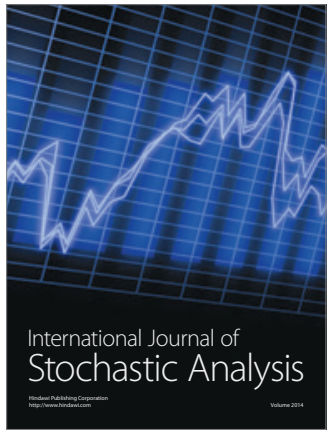

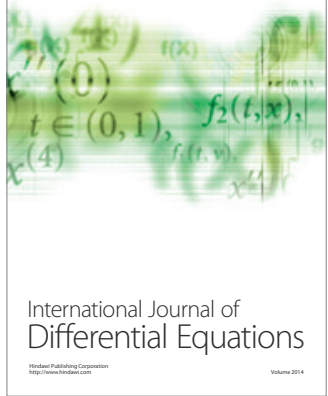
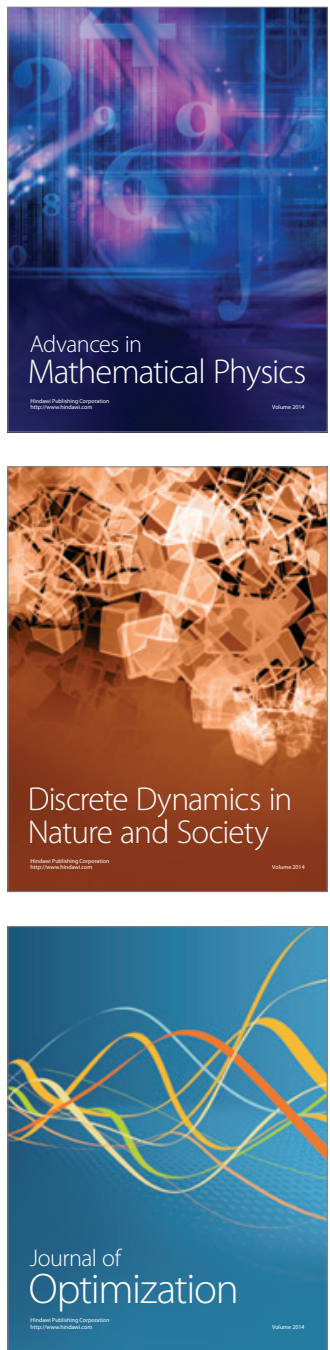